\title{
Uncertainty evaluation of a traceable dynamic force transfer standard
}

\author{
Saher Hassan ${ }^{1}$, Ch. Schlegel I , R. Kumme ${ }^{1}$, R. Tutsch ${ }^{2}$ \\ ${ }^{1}$ Physikalisch-Technische Bundesanstalt,Bundesallee 100, 38116 Braunschweig, \\ Germany \\ ${ }^{2}$ TU Braunschweig, Institut für Produktionsmesstechnik, Braunschweig, Germany \\ saher.r.hasan.ext@ptb.de
}

\begin{abstract}
A dynamic force transfer standard is designed at PTB to provide the traceability to dynamic testing metrology field. This contribution describes a method of evaluation the uncertainty of the primary dynamic force calibration of transfer standards, including the description of the uncertainty models of the sensitivity, which is the main measurand. Some influences have been reduced like the effect of the misalignment and telting effects. Other new contributions have been introduced like the contribution of the acceleration distribution correction due to elastic deformation of load masses, Sine fit, and vibration isolation of the laser vibrometer. A comparison between the estimation of uncertainty using classical GUM technique and Monte Carlo simulation has been performed.
\end{abstract}

Keywords: Dynamic force, Uncertainty, Dynamic force transfer standard.

\section{Introduction}

Dynamic force measurement plays a critical role in many industrial applications like automotive, aerospace, off shore energy, healthcare and construction. There is a need to convey traceability to this application in terms of dynamic force measurement. PTB has been developed a dynamic force transducer to act as a transfer standard for dynamic force metrology to provide the traceabililty to the dynamic force testing field. This transfer standard has been dynamically calibrated and metrologically characterised. The main tool of the metrological characterisation is the uncertainty the detailed dewscription of the dynamic calibration method has been mentioned in [1-3]. The error sources in dynamic force measurement and calibration are mentioned in [4-10].

\section{Mathematical model}

The dynamic sensitivity of a force measuring device can be expressed in terms of measurands as the ratio of the output signal of the force measuring device $U_{V}$ in $\mathrm{mV} / \mathrm{V}$ and the acting dynamic force $F_{d y n}$ in $\mathrm{N}$ with a magnitude of:

$$
S_{f}=\frac{U_{V}}{F_{d y n}}
$$

While the phase of sensitivity is determined as the phase difference between laser vibrometer, which corresponds to the reference force, and the output signal of the force measuring device.

$$
\Delta \varphi_{S_{f}}=\varphi_{F_{d y n}}-\varphi_{U_{V}}
$$

The output signal of the force measuring device $U_{V}$ is given by the ratio of the output signal of the bridge amplifier and the bridge excitation voltage

Using the excitation of an electrodynamic shaker, the dynamic force acting on the force transducer:

$$
F_{d y n}=\int_{V} \rho \cdot a(x, t) \cdot d V
$$

The distribution in acceleration $a(x, t)$ can be approximated by multiplying the acceleration $a_{t}$ by a frequency-dependent correction factor, $K_{o}$.

$$
K_{o}=\frac{\sin \left(\sqrt{k_{m} \omega^{2}} \cdot l\right)}{\sqrt{k_{m} \omega^{2}} \cdot l}, \quad k_{m}=\frac{\rho}{E}
$$

The acting dynamic force is then the product of all acting masses and their corresponding 
accelerations. In case of measurement of acceleration on the surface of the top mass, $a_{t}$, the acting dynamic force is calculated from.

$$
F_{d y n}=a_{t} \cdot\left(m_{t}+m_{i}\right) K_{o}
$$

The internal mass $m_{i}$ is determined using the equation

$$
S_{f}=\frac{U_{V}}{a_{t} \cdot\left(m_{t}+m_{i}\right)}
$$

That represents the dynamic sensitivity of the force proving instrument, to get the static sensitivity; $S_{f=0}$ we can rewrite the above equation like

$$
\left(\frac{U_{V}}{a_{t}}\right)_{f=0}=S_{f=0} \cdot\left(m_{t}+m_{i}\right)
$$

This lead to a linear equation between the ration of the transducer output to the top acceleration at one side and the unknown internal mass at the other side

$$
\left(\frac{U_{V}}{a_{t}}\right)_{f=0}=S_{f=0} \cdot m_{t}+S_{f=0} \cdot m_{i}
$$

With an offset equals $S_{f=0} \cdot m_{t}$ slope of $S_{f=0} \cdot m_{i}$. To get the variance of the internal mass as an influence to the uncertainty budget of the dynamic sensitivity

To calculate the dynamic sensitivity of the dynamic force measuring instrument, sinusoidal excitations at 17 different frequencies from 25 to $2000.25 \mathrm{~Hz}$ are performed. sine fitting is carried out by applying a least square fitting function of Matlab software. The uncertainty contribution of the fitting function is estimated using a Monte Carlo simulation that will be deployed in details in the next sections. The sampling rate was selected to be $25.625 \mathrm{kHz}$ that is more than ten times the maximum excitation frequency, which satisfies the NyquistShannon sampling theorem. At each frequency, a number of $2^{12}$ (4096) points are stored for each channel to avoid leakage error in the estimation of the sine function parameters. A number of a minimum of 10 cycles of data were recorded for each test frequency.

\section{Elimination of tilting and misalignment effects}

The acceleration of the mass measured by the laser vibrometer depends on the position of its laser beam on the top of the load mass. In this setup, 24 points are selected to make three octagons around each other. All three octagons are concentric to the load mass center as shown in Figure 1 above. Figure 1 below shows the acceleration distribution on top of the load mass at every octagon with different excitation frequencies. The more distance between measuring point and the load mass center, the higher variance in acceleration values exists. This is a combined resultant of the misalignment effect and the tilting modes. The variation of acceleration distribution on top of the load mass increases after resonance -around $1 \mathrm{kHz}$ of the applied load masses- due to the increasing effect of rocking modes within higher frequencies. To eliminate the effect of tilting and misalignment effects on the resulted uncertainty, an averaging of every two points against each other has been performed. The advantage of that averaging, that if the variation of the acceleration distribution is only because of the misalignment or even a tilting vibration mode of the load mass around its center of gravity, this will lead to a standard deviation of zero. Figure 1 below shows a case vibration where the variation of the acceleration distribution on top of the load mass comes only from the tilting of the load mass around its center of gravity. The averaging of each two acceleration vector the same $r$ coordinate and a shift of $\theta$ coordinate by $\pi$.
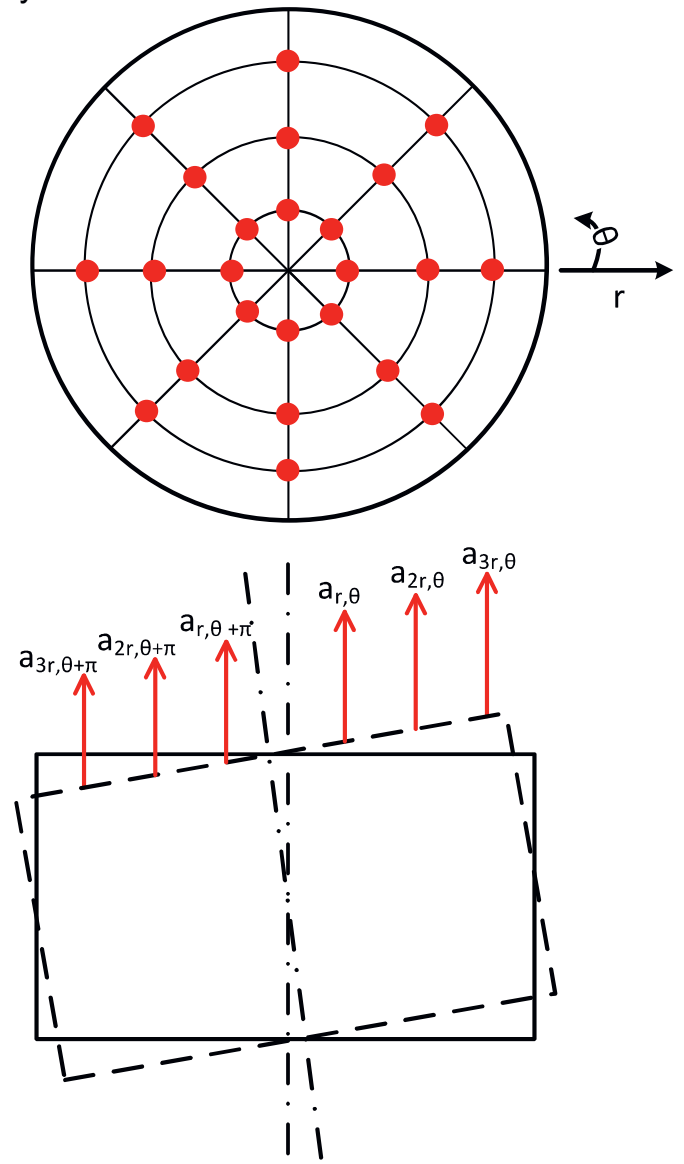
Figure 1 : Schematic of acceleration measurement on top of the load mass shows the 24 measurement points that are distributed to make three concentric octagons. top shows a top view and bottom shows section view with a dashed explanation of a mode shape that represents the rotation of the load mass around its center.

\section{Uncertainty Analysis}

The uncertainty of the sensitivity is determined as

$$
u_{S_{f}}^{2}=u_{U_{V}}^{2} \frac{\partial S_{f}^{2}}{\partial U_{V}}+u_{F_{d y n}}^{2} \frac{\partial S_{f}{ }^{2}}{\partial F_{d y n}}
$$

The quantity $\mathrm{u}_{r e p}$ is the uncertainty contribution due to the error of reproducibility of the force measuring device in addition to the effects of the misalignment of between the sensing axis of the force transducer and the principal axis of motion. The different uncertainty contributions to the dynamic sensitivity of force measuring devices are shown in Figure 2, the red contributions are neglected.

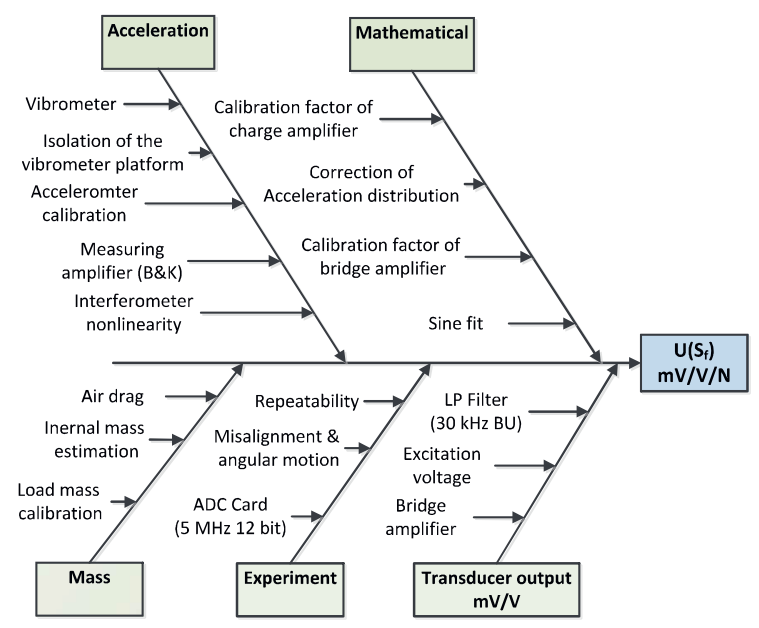

Figure 2: Ishikawa diagram of the uncertainty contributions to the dynamic sensitivity of force measuring devices.

Each suspected contribution to possible significant uncertainty is evaluated by measurement, estimation, by calculation or estimation by performing Monte Carlo MC simulation. The different predicted uncertainty contribution and their estimation method are listed in Table 1.

Table 1: Different uncertainty sources and corresponding estimation methods

\begin{tabular}{cc}
\hline $\begin{array}{c}\text { Uncertainty } \\
\text { Contribution }\end{array}$ & Evaluation Method \\
\hline Vibrometer & Calibration certificate \\
Bridge amplifier & Calibration certificate \\
Charge amplifier & Calibration certificate \\
Calculation of \\
Air Drag & empirical formula \\
Sine fit & MC simulation \\
ADC Card & MC simulation \\
Repeatability & Measurement \\
Correction of & MC simulation \\
acceleration & \\
distribution & Measurement \\
vibration of the & platform \\
Load mass & Measurement \\
\hline
\end{tabular}

\section{Uncertainty of voltage output}

The calibration of the bridge amplifier is performed with the precise multimeter HP3458A to calibrate the whole transfer function of the output signal of the force measuring device in $\mathrm{mV} / \mathrm{V}$ with a certain uncertainty, $\mathrm{u}_{U_{c a l}}$. The uncertainty of the output signal of the force measuring device is determined as

$$
u_{U_{V}}^{2}=u_{U_{\text {cal }}}^{2}+u_{\text {filt }}^{2}+u_{\text {rep }}^{2}+u_{\text {sine }}^{2}
$$

while $\mathrm{u}_{\text {filt }}$ is the uncertainty contribution of the used low pass filter, a Butterworth low pass filter has been inserted with a $30 \mathrm{kHz}$ cut-off frequency.

\section{Uncertainty of low-pass filter}

A Butterworth low-pass filter with a $30 \mathrm{kHz}$ and $100 \mathrm{kHz}$ cut-off frequency has been applied to the bridge amplifier and charge amplifier respectively. The sampling rate of the data aquisition is set to $25.6 \mathrm{kHz}$, this offer a maximum attenuation at the Nyquist frequency. The Butterworth filter is a special type of socalled Chebyshev filter with the amplitude frequency response $G_{n}(\omega)$ as

$$
G_{n}(\omega)=\left|H_{n}(j \omega)\right|=\frac{1}{\sqrt{1+\varepsilon^{2} T_{n}^{2}\left(\frac{\omega}{\omega_{0}}\right)}}
$$

Where $\varepsilon$ is the ripple factor, $\omega_{0}$ is the cut-off frequency and $T_{n}$ is a Chebyshev polynomial of the $\mathrm{n}$ order. With the application of the Butterworth filter, a ripple is set to $0 \%$ which leads to a zero amplitude loss at the maximum test frequency of $2 \mathrm{kHz}$. 


\section{Calibration factor of charge and bridge amplifiers}

The calibration the bridge and charge amplifiers of that have been used to measure force and acceleration signals respectively is performed with the same excitation frequencies that have been applied to calibrate the force transducer. The correction of the amplitude and phase frequency response of both amplifiers is performed point by point without applying curve fitting.

\section{Uncertainty of sine-fit approximation}

A Least-square fit is used to estimate the magnitude and phase of the measurands. The error of the estimate is affected by the resolution and sampling time of the ADC card in addition to the residual error of the sine approximation itself. To estimate the uncertainty of the sine approximation, a Monte Carlo simulation was set to run $\mathrm{M}=1 \times 10^{5}$ trials of the model:

$$
A=A_{0} \sin (\omega t+\varphi)
$$

\section{Uncertainty of acting dynamic force}

This has an uncertainty of

$$
\begin{aligned}
u_{F_{d y n}}^{2}=u_{a_{t}}^{2} \frac{\partial F_{d y n}{ }^{2}}{\partial a_{t}} & +u_{m_{t}}^{2} \frac{\partial F_{d y n}{ }^{2}}{\partial m_{t}} \\
& +u_{m_{i}}^{2} \frac{\partial F_{d y n}{ }^{2}}{\partial m_{i}} \\
& +u_{K_{o}}^{2} \frac{\partial F_{d y n}{ }^{2}}{\partial K_{t o}} \\
& +u_{d r a g}^{2} \frac{\partial F_{d y n}}{\partial F_{d r a g}}
\end{aligned}
$$

The acting load masses include the top mass $m_{t}$ and the effective internal mass of the force transducer $m_{i}$ that applies a dynamic force on the sensing transducer element. In the case of measurement of acceleration in the force transducer close to the transducer sensing element, $\mathrm{a}_{\mathrm{b}}$, the acting dynamic force will be

$$
F_{d y n}=a_{t} \cdot m_{t} K_{o}+a_{b} \cdot m_{i} \text {. }
$$

With uncertainty of

$$
\begin{aligned}
u_{F_{d y n}}^{2}=u_{a_{t}}^{2} \frac{\partial F_{d y n}{ }^{2}}{\partial a_{t}} & +u_{a_{b}}^{2} \frac{\partial F_{d y n}{ }^{2}}{\partial a_{b}} \\
& +u_{m_{t}}^{2} \frac{\partial F_{d y n}{ }^{2}}{\partial m_{t}} \\
& +u_{m_{i}}^{2} \frac{\partial F_{d y n}{ }^{2}}{\partial m_{i}} \\
& +u_{K_{o}}^{2} \frac{\partial F_{d y n}}{\partial K_{t o}} \\
& +u_{d r a g}^{2} \frac{\partial F_{d y n}}{\partial F_{d r a g}}
\end{aligned}
$$

\section{Uncertainty of acceleration distribution factor, $\mathbf{K}_{\mathbf{o}}$}

The uncertainty of the correction factor of acceleration distribution $\mathrm{K}_{\mathrm{o}}$ is dominated by uncertainty in the material properties, which were taken from tabulated data. A Monte Carlo simulation has been used to calculate the uncertainty using the simulation of the variation of material properties.

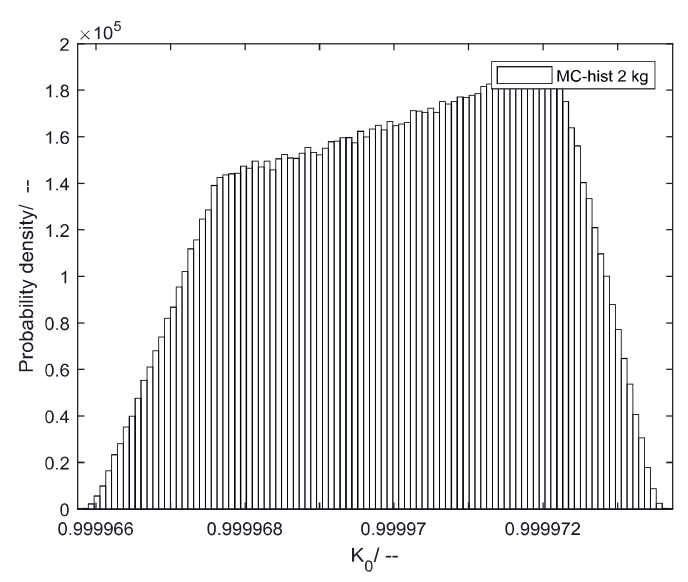

Figure 3: Output PDFs of the acceleration distribution factor $\mathrm{KO}$ for 2 load masses with variation of $3^{*} 10-495 \%$ confidence level.

\section{Uncertainty of air drag}

As the load mass moves, a parasitic drag force acts on its surface due to the resistance of air. This force can be estimated from the load mass geometry, the maximum velocity of the load mass, $\mathrm{V}$.

$$
\mathrm{D}=0.5 \mathrm{C}_{\mathrm{d}} \rho \mathrm{V}^{2} \mathrm{~A} .
$$

Where $D$ is the drag force, $\rho$ is the density of air, $A$ is the area of the load mass that subjected to air resistance, and $C_{d}$ is the drag coefficient based on empirical data [11]. At the highest velocities (approximately $1 \mathrm{~m} \mathrm{~s}^{-1}$ ), the total drag force is estimated to be below 0.5 $\mathrm{mN}$. The drag force is not corrected for, but rather is treated as an uncertainty of magnitude equal to the maximum expected drag of $0.5 \mathrm{mN}$. A uniform distribution is assumed for this quantity.

\section{Uncertainty of load and internal masses}

The mass value of the load mass was determined with a relative uncertainty of $5 \cdot 10^{-3}$ $\%$. To estimate the uncertainty of the internal mass determination, an MC simulation is deployed to simulate the linear equation taking 
into consideration a uniform distribution of all fit error in the estimation of the parameters of the above equation.

\section{Uncertainty of vibration isolation}

As the shaker is the source of the required vibration, its vibration might be transmitted to the vibrometer platform. An active and passive damping base is installed to the base of the laser head to isolate vibration that comes from the shaker or even from earth. A vibration transmission coefficient has been experimentally measured. The transmission function was measured using accelerometers mounted in both locations; on top of the shaker armature and the frame of the laser vibrometer. Figure 4Error! Reference source not found. shows the measurements of the transmission coefficient with the dimpling facilities of the vibrometer platform. The results show the neglected effect of the shaker vibration behind $1 \mathrm{kHz}$. The uncertainty contribution after $1 \mathrm{kHz}$ was taken to be $0.06 \%$.

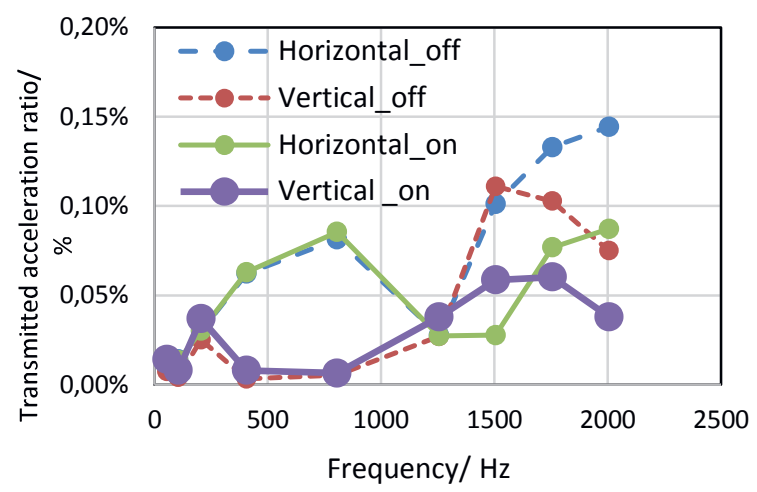

Figure 4: : The vibration transmission ration between the acceleration measured on top of the shaker armature and the acceleration of the frame of the laser vibrometer.

\section{Uncertainty of Phase measurement}

The uncertainty $U_{\text {phase }}$ in the measured transducer sensitivity phase is calculated according to

$$
u_{\text {phase }}^{2}=u_{\text {ph,acc }}^{2}+u_{\text {ph,amp }}^{2}+u_{\text {ph,rep }}^{2} .
$$

The quantity $U_{p h, a c c}$ is the uncertainty due to the phase delay introduced by the acceleration measurement which results mainly from the sampling rate of the data acquisition card of the laser vibrometer and was estimated using the calibration of the laser vibrometer with an expanded uncertainty of $\pm 0.3^{\circ}$. The uncertainty
$\mathrm{U}_{\mathrm{ph}, \mathrm{amp}}$ accounts for the uncertainty contribution of the bridge amplifier which contributes with an expanded uncertainty of $\pm 0.2^{\circ}$. The uncertainty $U_{\mathrm{ph}, \mathrm{rep}}$ describes the repeatability of the phase measurement of the force transducer itself. A summary of the sensitivity magnitude and phase measurement uncertainties is presented in Table 2 and Table 3.

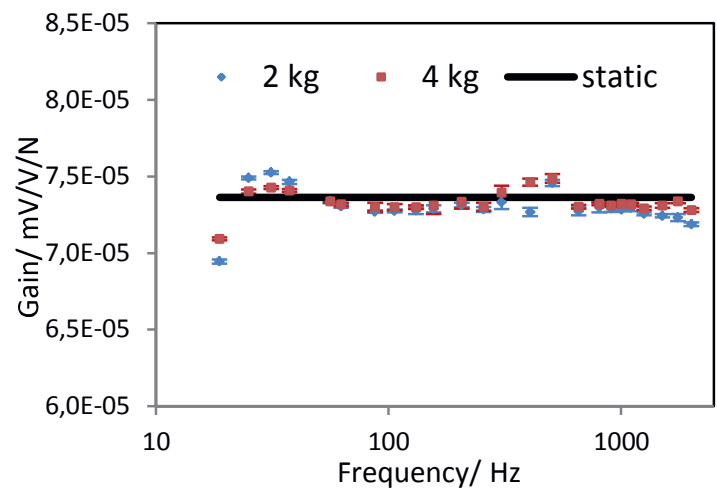

Figure 5: The measured sensitivity and the corresponding relative expanded uncertainty using different load masses in comparison to the static sensitivity

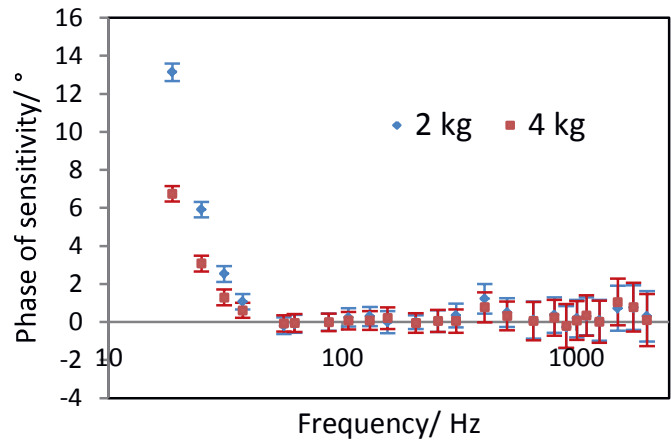

Figure 6: Phase of the sensitivity of the transducer and the corresponding expanded uncertainty $(k=2)$

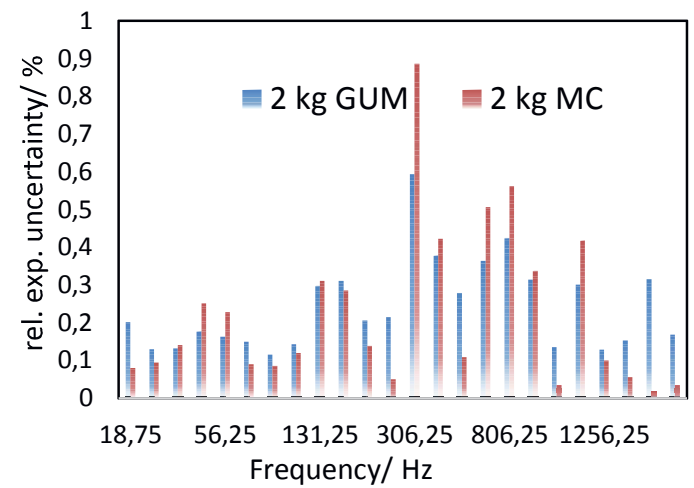


Figure 7: Comparison between the relative expanded uncertainty using classical GUM approach and the 95\% coverage interval of the output sensitivity using the $M C$ approach.

Table 2: Summary of uncertainties associated with force transducer sensitivity

\begin{tabular}{|c|c|c|c|}
\hline Source & \multicolumn{2}{|c|}{$\begin{array}{c}\text { Uncertainty } \\
\text { contribution } \\
{[\mathrm{mV} / \mathrm{V} / \mathrm{N}]}\end{array}$} & $\begin{array}{c}100 \times \\
\text { Inde } \\
x \\
\end{array}$ \\
\hline \multicolumn{4}{|c|}{ 1. Uncertainty of applied force, $u_{F_{d y n}}$} \\
\hline $\begin{array}{l}\text { Vibrometer } \\
\text { calibration, } \boldsymbol{u}_{\boldsymbol{a}_{t} \boldsymbol{r} \boldsymbol{e f}}\end{array}$ & \multicolumn{2}{|c|}{$\begin{array}{l}3,06 \times 10^{-08} \\
3,51 \times 10^{-08}\end{array}$} & $9-40$ \\
\hline $\begin{array}{l}\text { Vibrometer } \\
\text { repeatability, } \boldsymbol{u}_{\boldsymbol{a}_{\boldsymbol{t}} \boldsymbol{r e p}}\end{array}$ & \multicolumn{2}{|c|}{$\begin{array}{l}1,54 \times 10^{-09} \\
2,66 \times 10^{-07}\end{array}$} & $2-68$ \\
\hline $\begin{array}{l}\text { Vibration isolation, } \\
\boldsymbol{u}_{\boldsymbol{a}_{\boldsymbol{t}} \text { iso }}\end{array}$ & \multicolumn{2}{|c|}{$0-2,42 \times 10^{-08}$} & $0-22$ \\
\hline $\begin{array}{l}\text { Accelerometer } \\
\text { calibration, } \boldsymbol{u}_{\boldsymbol{a}_{\boldsymbol{b}} \boldsymbol{r e f}}\end{array}$ & \multicolumn{2}{|c|}{$\begin{array}{l}2,46 \times 10^{-09} \\
1,03 \times 10^{-08}\end{array}$} & $1-10$ \\
\hline $\begin{array}{l}\text { Accelerometer } \\
\text { repeatability, } \boldsymbol{u}_{a_{b} r e p}\end{array}$ & \multicolumn{2}{|c|}{$\begin{array}{l}1,99 \times 10^{-10} \\
5,17 \times 10^{-09}\end{array}$} & $0.2-2$ \\
\hline Top mass, $\boldsymbol{u}_{\boldsymbol{m}_{t}}$ & \multicolumn{2}{|c|}{$\begin{array}{l}3,06 \times 10^{-09} \\
3,56 \times 10^{-09}\end{array}$} & $1-4$ \\
\hline $\begin{array}{l}\text { Sine approximation, } \\
\boldsymbol{u}_{\text {sine }}\end{array}$ & \multicolumn{2}{|c|}{$\begin{array}{l}2,11 \times 10^{-13}- \\
2,46 \times 10^{-13}\end{array}$} & $\begin{array}{l}< \\
0.02\end{array}$ \\
\hline Internal mass, $\boldsymbol{u}_{\boldsymbol{m}_{\boldsymbol{i}}}$ & \multicolumn{2}{|c|}{$\begin{array}{l}3,23 \times 10^{-11} \\
1,76 \times 10^{-10}\end{array}$} & $0-0.2$ \\
\hline Air drag, $\boldsymbol{u}_{d r a g}$ & \multicolumn{2}{|c|}{$\begin{array}{l}3,64 \times 10^{-12} \\
9,64 \times 10^{-11}\end{array}$} & $0-0.1$ \\
\hline $\begin{array}{l}\text { Acceleration } \\
\text { distribution, } \boldsymbol{u}_{\boldsymbol{K}_{o}}\end{array}$ & \multicolumn{2}{|c|}{$\begin{array}{l}5,45 \times 10^{-14}- \\
2,40 \times 10^{-13}\end{array}$} & $\begin{array}{l}< \\
0.02 \\
\end{array}$ \\
\hline \multicolumn{4}{|c|}{ 2.Uncertainty of Voltage output, $u_{U_{V}}$} \\
\hline $\begin{array}{l}\text { Bridge amplifier, } \\
\boldsymbol{u}_{\boldsymbol{U}_{\boldsymbol{V}} \boldsymbol{r} \boldsymbol{e f}}\end{array}$ & \multicolumn{2}{|c|}{$\begin{array}{l}1,74 \times 10^{-08} \\
1,88 \times 10^{-08}\end{array}$} & $5-22$ \\
\hline $\begin{array}{l}\text { Transducer } \\
\text { Repeatability, } \\
\boldsymbol{u}_{\boldsymbol{U}_{\boldsymbol{V}} \boldsymbol{r e p}}\end{array}$ & \multicolumn{2}{|c|}{$\begin{array}{l}6,24 \times 10^{-09} \\
2,13 \times 10^{-07}\end{array}$} & $7-71$ \\
\hline \multicolumn{2}{|c|}{$\begin{array}{l}\text { Rel. combined standard } \\
\text { uncertainty, } \mathrm{u}_{\mathrm{S}_{\mathrm{Vf}}}\end{array}$} & \multicolumn{2}{|c|}{$\begin{array}{l}5,76 \times 10-04- \\
3,79 \times 10-03\end{array}$} \\
\hline \multicolumn{2}{|c|}{$\begin{array}{l}\text { Rel. expanded uncertainty, } \\
\mathrm{U}_{\mathrm{S}_{\mathrm{Vf}}}(\mathrm{k}=2)\end{array}$} & \multicolumn{2}{|c|}{$\begin{array}{l}1,15 \times 10-03- \\
7,57 \times 10-03\end{array}$} \\
\hline
\end{tabular}

Table 3: Summary of uncertainties associated with phase measurement

\begin{tabular}{|c|c|c|}
\hline Source & $\begin{array}{c}\text { Uncertaint } \\
\mathrm{y}\end{array}$ & $\begin{array}{c}100 \times \mathrm{I} \\
\mathrm{ndex}\end{array}$ \\
\hline
\end{tabular}

\begin{tabular}{|l|c|c|}
\hline & $\begin{array}{c}\text { contributio } \\
\mathrm{n}\left[^{\circ}\right]\end{array}$ & \\
\hline Acceleration, $\mathrm{u}_{\mathrm{ph}, \mathrm{acc}}$ & 0.3 & $22-58$ \\
\hline $\begin{array}{l}\text { Bridge amplifier, } \\
\mathrm{u}_{\text {ph,amp }}\end{array}$ & 0.2 & $15-39$ \\
\hline $\begin{array}{l}\text { Repeatability, } \\
\mathrm{u}_{\text {ph,rep }}\end{array}$ & $0.03-2$ & $3-64$ \\
\hline $\begin{array}{l}\text { Rel. combined standard } \\
\text { uncertainty, } \mathbf{u}_{\text {phase }}\end{array}$ & $0,18-0,36$ \\
\hline $\begin{array}{l}\text { Rel. expanded uncertainty, } \\
\mathbf{U}_{\text {phase }}(\boldsymbol{k}=\mathbf{2})\end{array}$ & $0,68-1,36$ \\
\hline
\end{tabular}

\section{Conclusion}

This paper describes a detailed uncertainty model for the metrological characterization of dynamic force transfer standards in their calibration against dynamic force primary standard. Many uncertainty sources have been reduced like tilting and misalignment effects. Other contributions have been developed like the correction of the acceleration distribution over load masses, and sine-fit approximation. A good agreement has been observed between the two uncertainty estimation approaches; Classical GUM approach and MC approach.

\section{Acknowledgement}

The authors gratefully acknowledge support of the Braunschweig International Graduate School of Metrology (B-IGSM).

\section{References}

[1] Schlegel C, Kieckenap G, Glöckner B, Buß $A$ and Kumme R 2012 Traceable periodic force calibration Metrologia 49 224-35

[2] Vlajic N and Chijioke A 2016 Traceable dynamic calibration of force transducers by primary means Metrologia 53 S136-48

[3] Medina N and de Vicente J 2014 Force sensor characterization under sinusoidal excitations Sensors (Switzerland) 14 18454-73

[4] Sawla A 1991 Measurment of dynamic forces and compensation of errors in fatigue testing Proc. 12th IMEKO World Congress (Beijing, China) pp 5-10

[5] Elster C and Link A 2008 Uncertainty evaluation for dynamic measurements modelled by a linear time-invariant system Metrologia 45 464-73

[6] Kumme R 1994 Error sources in dynamic force calibration Proc. 13th IMEKO World Congress (Torino, Italy) pp 259-64

[7] Ch. Schlegel, G. Kiekenap and R K 2014 Uncertainty contributions in sinusoidal force measurement IMEKO 22nd TC3, 12th TC5 and 3rd TC 22 International Conference (Cape Town, Republic of South Africa) pp $1-4$ 
[8] Zhang L, Wang Y, Yin X, Schlegel C and Kumme R 2018 Comparison results between PTB and CIMM on the force transducer calibration under sinusoidal loading J. Phys. Conf. Ser. 1065042019

[9] Schlegel C, Kiekenap G and Kumme R 2015 Uncertainty evaluation of sinusoidal force measurement Proc. 21st IMEKO World Congress (Prague)

[10] Kumme R 1997 The main influences on the dynamic properties of force measuring devices Proc. 14th IMEKO World Congress (Tampere, Finland) pp 102-7

[11] Hoener S F 1965 Fluid Dynamics Drag: Practical Information on Aerodynamic Drag and Hydrodynamic Resistance 